\title{
Oseltamivir Ring Prophylaxis for Containment of 2009 H1N1 Influenza Outbreaks
}

\author{
Vernon J. Lee, M.B., B.S., M.P.H., Jonathan Yap, M.B., B.S., Alex R. Cook, Ph.D., \\ Mark I. Chen, M.B., B.S., Ph.D., Joshua K. Tay, M.B., B.S., Boon Huan Tan, Ph.D., \\ Jin Phang Loh, M.Sc., Seok Wei Chew, B.Sc., Wee Hong Koh, B.Sc., \\ Raymond Lin, M.B., B.S., Lin Cui, Ph.D., Charlie W.H. Lee, M.Sc., \\ Wing-Kin Sung, Ph.D., Christopher W. Wong, Ph.D., Martin L. Hibberd, Ph.D., \\ Wee Lee Kang, M.B., B.S., M.Med., Benjamin Seet, M.B., B.S., M.P.H., \\ and Paul A. Tambyah, M.D.
}

ABSTRACT

From the Biodefence Centre, Ministry of Defence (V.J.L., J.Y., J.K.T.); the Centre for Health Services Research (V.J.L.), the Department of Epidemiology and Public Health (V.J.L., M.I.C.), the Department of Statistics and Applied Probability (A.R.C.), and the Division of Infectious Diseases (P.A.T.), National University of Singapore; the Department of Clinical Epidemiology, Tan Tock Seng Hospital (M.I.C.); the Defence Medical and Environmental Research Institute, DSO National Laboratories (B.H.T., J.P.L., S.W.C., W.H.K.); the National Public Health Laboratory, Ministry of Health (R.L., L.C.); the Genome Institute of Singapore (C.W.H.L., W.-K.S., C.W.W., M.L.H.); and the Headquarters Medical Corps, Singapore Armed Forces (W.L.K., B.S.) - all in Singapore; and the National Centre for Epidemiology and Population Health, Australian National University, Canberra, ACT, Australia (V.J.L.). Address reprint requests to Dr. V.J. Lee at 42 How Sun Dr., 538611 Singapore, Singapore, or at vernonljm@hotmail.com.

N Engl J Med 2010;362:2166-74.

Copyright (C) 2010 Massachusetts Medical Society.
BACKGROUND

From June 22 through June 25, 2009, four outbreaks of infection with the pandemic influenza A (H1N1) virus occurred in Singapore military camps. We report the efficacy of ring chemoprophylaxis (geographically targeted containment by means of prophylaxis) with oseltamivir to control outbreaks of 2009 H1N1 influenza in semiclosed environments.

\section{METHODS}

All personnel with suspected infection were tested and clinically isolated if infection was confirmed. In addition, we administered postexposure ring chemoprophylaxis with oseltamivir and segregated the affected military units to contain the spread of the virus. All personnel were screened three times weekly both for virologic infection, by means of nasopharyngeal swabs and reverse-transcriptase-polymerase-chain-reaction assay with sequencing, and for clinical symptoms, by means of questionnaires.

\section{RESULTS}

A total of 1175 personnel were at risk across the four sites, with 1100 receiving oseltamivir prophylaxis. A total of 75 personnel $(6.4 \%)$ were infected before the intervention, and $7(0.6 \%)$ after the intervention. There was a significant reduction in the overall reproductive number (the number of new cases attributable to the index case), from 1.91 ( $95 \%$ credible interval, 1.50 to 2.36 ) before the intervention to 0.11 ( $95 \%$ credible interval, 0.05 to 0.20 ) after the intervention. Three of the four outbreaks showed a significant reduction in the rate of infection after the intervention. Molecular analysis revealed that all four outbreaks were derived from the New York lineage of the $2009 \mathrm{H} 1 \mathrm{~N} 1$ virus and that cases within each outbreak were due to transmission rather than unrelated episodes of infection. Of the 816 personnel treated with oseltamivir who were surveyed, 63 (7.7\%) reported mild, nonrespiratory side effects of the drug, with no severe adverse events.

CONCLUSIONS

Oseltamivir ring chemoprophylaxis, together with prompt identification and isolation of infected personnel, was effective in reducing the impact of outbreaks of 2009 H1N1 influenza in semiclosed settings. 
T HE 2009 PANDEMIC INFLUENZA A (H1N1) virus has spread rapidly worldwide, despite initial attempts at containment through screening, isolation, and quarantine..$^{1-3}$ Many countries moved rapidly into the mitigation phase after the outbreak was detected, which affected essential services, especially in the health and education sectors. Mexico, the first country affected, shut down all major public services for a week to halt transmission of the virus. Other large outbreaks in population centers had a similar effect on essential services. Even though pandemic vaccines are available, the lack of availability during a pandemic results in incomplete global protection.

Mathematical models of the efficacy of containment measures in an influenza epicenter have been described, ${ }^{4,5}$ although these measures ultimately proved ineffective at preventing the spread of the 2009 pandemic H1N1 virus. However, containment measures may be effective within specific closed environments, such as schools, health care settings, or military installations, all of which have a high risk of transmission. ${ }^{6}$ Chemoprophylaxis with a neuraminidase inhibitor has been effective in preventing the household transmission of influenza, ${ }^{7}$ and modeling studies have predicted that well-timed chemoprophylaxis could significantly reduce the rate of absenteeism among health care workers due to illness, to maintain business continuity. ${ }^{8}$

Although antiviral "ring chemoprophylaxis" strategies (aimed at geographically targeted containment by means of prophylaxis) were predicted to be effective in mathematical models, data are needed to document their actual effectiveness during a pandemic. We therefore describe our experience in responding to four outbreaks of the 2009 pandemic influenza A (H1N1) virus in military camps (including one in a health care setting) and evaluate the role of oseltamivir "ring chemoprophylaxis" in attenuating transmission of the virus.

\section{METHODS}

Singapore is a city-state of 4.84 million people. ${ }^{9}$ All Singaporean men perform 2 years of military service after high school, at 18 to 19 years of age. Most military personnel live in barracks-style accommodations on weekdays and return home on weekends, resulting in an interaction between the military community and the Singapore population.

Singapore identified its first imported case of infection with the 2009 pandemic influenza A (H1N1) virus on May 27, 2009, ${ }^{10}$ and the first transmission to the local community was reported on June 18, 2009. ${ }^{11}$ In line with World Health Organization (WHO) recommendations, ${ }^{12}$ Singapore began the transition to mitigation on July 1 , 2009. ${ }^{13}$ The Singapore Armed Forces (SAF) identified its first imported case of infection on June 15,2009 , and its first four outbreak clusters (outbreaks I, II, III, and IV) involving local transmission from June 22 to 25, 2009.

\section{NATIONAL PROTOCOLS AND MANAGEMENT}

A suspected case of 2009 H1N1 influenza was defined as influenza-like illness (temperature $\geq 38.0^{\circ} \mathrm{C}$ with cough or sore throat) with an onset of symptoms within 7 days after travel to an affected area, close contact with a person with confirmed infection, or contact with a local cluster of infected persons. ${ }^{14}$ Laboratory confirmation of suspected cases was performed by means of real-time reverse-transcriptase-polymerase-chain-reaction (RT-PCR) assay or viral culture. ${ }^{14}$

Until July 1, 2009, all persons with suspected infection with the $2009 \mathrm{H} 1 \mathrm{~N} 1$ virus were screened with the use of RT-PCR assay, according to national protocols, ${ }^{15}$ and patients with confirmed infection were isolated in hospitals to prevent transmission. Contact tracing was performed to identify close contacts, defined as persons who had had unprotected exposure, within $2 \mathrm{~m}$, to an infected patient for 1 hour or more since the day before the onset of symptoms. ${ }^{10}$ Most contacts were quarantined at home for a 7-day period.

\section{SAF PROTOCOL AND MANAGEMENT}

Performing its function as a critical national resource, the SAF implemented additional interventions to contain the spread of the 2009 H1N1 virus. Primarily, "ring prophylaxis" with oseltamivir (Tamiflu, Roche), at a dose of $75 \mathrm{mg}$ daily, was administered to coworkers of the patient with confirmed infection for a period of 10 days after exposure. ${ }^{16}$ The oseltamivir had been purchased and stockpiled several years previously as part of the SAF influenza-pandemic preparedness plan. A coworker was defined as a member of the same military unit, where contact opportunities were substantial even if they did not fulfill the Singapore Ministry of Health criteria for close contact. This wider definition was prompted by difficulties in identifying actual contacts and the 
practicalities of rapidly administering prophylaxis. Larger prophylaxis rings were instituted if cases were present in multiple units. In addition, interactions between affected units and other units were reduced within the camp, by allocating to each unit different times of arrival, departure, and meal delivery.

\section{EPIDEMIOLOGIC INVESTIGATION}

Our investigation of the outbreaks was approved by the SAF Joint Medical Committee, as well as the National University of Singapore and the Australian National University institutional review boards. Written informed consent was obtained from all persons for whom follow-up nasopharyngeal swabs were obtained, and oral assent was provided by all others during the surveys.

The four outbreaks occurred in different locations: one in each of three military units and one at a camp medical center. All personnel with suspected infection were tested and isolated in the hospital if the test was positive. In addition, all asymptomatic personnel in the same unit were screened through the collection of nasopharyngeal swabs, three times a week, to detect subclinical infections. ${ }^{17} \mathrm{~A}$ written questionnaire was administered at each screening visit, as well as after the completion of prophylaxis, to collect data on demographic characteristics, medical history, activity patterns, and clinical symptoms. Screening was performed until no additional cases were identified for 3 days after the last previously identified case or after the end of the 10-day prophylaxis period, whichever was later. After the prophylaxis period, a telephone questionnaire was administered to personnel who had left camp before the screening was completed.

\section{MOLECULAR DIAGNOSIS AND SEQUENCING}

Nasopharyngeal swabs were collected, resuspended in $2.0 \mathrm{ml}$ of viral-transport medium, and sent for RT-PCR testing, all within a 24-hour period. The RT-PCR assay involved protocols with the swine H1 forward-reverse primer set and probe. ${ }^{18}$ Positive samples with sufficient RNA underwent wholegenome sequencing according to a previously reported approach. ${ }^{19}$ The resulting sequences were used to generate phylogenetic trees with the use of Molecular Evolutionary Genetics Analysis 4 software. ${ }^{20}$ All sequenced samples were screened for known and suspected mutations that would confer oseltamivir resistance, including the H274Y mutation. Additional methods are described in the Supplementary Appendix (available with the full text of this article at NEJM.org).

\section{STATISTICAL ANALYSIS}

Following the statistical argument of Cauchemez and colleagues, ${ }^{21}$ we assumed that each case of 2009 H1N1 influenza leads to new cases, distributed as a Poisson variate with a mean of $\lambda$ or $\lambda \theta$ in the absence or presence of intervention, respectively, as well as a specific form for the generation interval. The $\lambda$ variable represents the reproductive number (the mean number of new cases attributable to the index case) in the absence of intervention, and $\lambda \theta$ the reproductive number after intervention. Analysis was performed according to the Bayesian paradigm, ${ }^{22}$ and with the use of the statistical programming language $\mathrm{R} .{ }^{23}$ The Supplementary Appendix describes that analysis as well as the methods used to quantify the strength of the intervention effect, obtain credible intervals, and evaluate the hypothesis of a reduction in infection rates after intervention (i.e., $\theta<1)$. For measures of statistical significance, we report the posterior hypothesis probabilities as described in the Supplementary Appendix.

\section{RESULTS}

A total of 82 confirmed cases of infection with the 2009 pandemic influenza A (H1N1) virus were identified during the four outbreaks (Table 1).

\section{OUTBREAK 1}

From June 21 to 22, 2009, four personnel (B, C, E, and F in Fig. 1) tested positive for 2009 H1N1 influenza. Three (B, E, and F) had performed overnight guard duty together on June 18, 2009. Four more $(\mathrm{A}, \mathrm{G}, \mathrm{H}$, and $\mathrm{I})$ were confirmed to be infected during initial investigations. The remaining 208 coworkers were given oseltamivir prophylaxis; of these, 81 were identified as close contacts and were quarantined at home. During the outbreak, three more personnel tested positive, of whom two (D and J) had not been initially identified as close contacts. Of the remaining 205 personnel, 185 (90.2\%) completed the course of prophylaxis. Fourteen personnel reported minor respiratory symptoms; 11 tested negative for $2009 \mathrm{H} 1 \mathrm{~N} 1$ influenza and 3 were not tested. The other personnel continued working in the camp, and none tested positive, as assessed by testing three consecutive na- 


\begin{tabular}{|c|c|c|c|c|c|}
\hline Variable & Total & Outbreak 1 & Outbreak 2 & Outbreak 3 & Outbreak 4 \\
\hline Total no. of personnel & 1175 & 216 & 47 & 219 & 693 \\
\hline Confirmed cases - no. (\%) & $82(7.0)$ & $11(5.1)$ & $6(12.8)$ & $2(0.9)$ & $63(9.1)$ \\
\hline Before intervention - no. (\%) & $75(6.4)$ & $8(3.7)$ & $6(12.8)$ & $2(0.9)$ & $59(8.5)$ \\
\hline After intervention — no. (\%) & $7(0.6)$ & $3(1.4)$ & 0 & 0 & $4(0.6)$ \\
\hline Posterior hypothesis probability & $<0.001$ & 0.11 & $<0.001$ & $<0.001$ & $<0.001$ \\
\hline \multicolumn{6}{|l|}{ Symptomatic personnel (excluding confirmed cases) } \\
\hline Tested and negative — no. (\%) & $23(2.0)$ & $11(5.1)$ & 0 & $1(0.5)$ & $11(1.6)$ \\
\hline Not tested - no. (\%) & $47(4.0)$ & $3(1.4)$ & 0 & $4(1.8)$ & $40(5.8)$ \\
\hline Mild respiratory symptoms only & $40(3.4)$ & $1(0.5)$ & 0 & $4(1.8)$ & $35(5.1)$ \\
\hline Reported fever with respiratory symptoms & $7(0.6)$ & $2(0.9)$ & 0 & 0 & $5(0.7)$ \\
\hline $\begin{array}{l}\text { Completion of oseltamivir prophylaxis - no./total no. } \\
(\%) \dagger\end{array}$ & $929 / 974(95.4)$ & $185 / 205(90.2)$ & $41 / 41(100)$ & $186 / 193(96.4)$ & $517 / 535(96.6)$ \\
\hline \multicolumn{6}{|l|}{$\begin{array}{l}\text { Confirmed cases and symptomatic personnel who } \\
\text { were not tested }\end{array}$} \\
\hline Total — no./total no. & $115 / 1161$ & $14 / 216$ & $6 / 47$ & $5 / 218$ & $90 / 680$ \\
\hline Before intervention - no./total no. (\%) & $85 / 1161(7.3)$ & $10 / 216(4.6)$ & $6 / 47(12.8)$ & $3 / 218(1.4)$ & $66 / 680(9.7)$ \\
\hline After intervention — no./total no. (\%) & $30 / 1076(2.8)$ & $4 / 206(1.9)$ & 0 & $2 / 215(0.9)$ & $24 / 614(3.9)$ \\
\hline Posterior hypothesis probability & $<0.001$ & 0.02 & $<0.001$ & 0.09 & $<0.001$ \\
\hline
\end{tabular}

* The posterior hypothesis probabilities were calculated for the comparison of the incidence of infection before intervention and after intervention, as described in the Supplementary Appendix.

$\dagger$ The number of subjects who completed the oseltamivir prophylaxis regimen excludes those with confirmed infections and those who could not be contacted.

$\lceil$ The number of confirmed cases and symptomatic personnel who were not tested excludes 14 symptomatic personnel who could not remember the date of onset of their illness. The percentage of confirmed cases and symptomatic personnel who were not tested before intervention is based on the total number with data; the percentage after intervention is based on the total number with data minus the number identified before intervention.

sopharyngeal swabs obtained over a 1-week period. Overall, 11 of the 216 personnel (5.1\%) were infected (Fig. 1).

\section{OUTBREAK 2}

In a military medical center, 6 of 47 health care workers tested positive from June 24 to 25, 2009. Because health care workers were essential for the medical center to function, oseltamivir prophylaxis was administered to the remaining 41 personnel, who continued to work while wearing personal protective equipment (N95 mask, gloves, gown, and cap). All 41 health care workers completed the prophylaxis, and none had evidence of infection on testing of three consecutive nasopharyngeal swabs obtained over a 1 -week period.

\section{OUTBREAK 3}

On June 23, 2009, the index patient presented with influenza-like illness and tested positive. On June
20, 2009, he had visited a nightclub in Singapore (where there was a separate outbreak). ${ }^{24}$ One other asymptomatic case in the unit was confirmed during initial investigations. The remaining 217 personnel in the unit were immediately started on prophylaxis, and active surveillance was performed, consisting of testing of two nasopharyngeal swabs obtained over a 3-day period. None tested positive. After prophylaxis, telephone surveillance was performed, with 193 of the 217 personnel (88.9\%) successfully contacted; 186 of 193 (96.4\%) had completed the prophylaxis. Only one soldier reported fever; he tested negative.

\section{OUTBREAK 4}

A unit of 693 army-reserve personnel entered the camp from the community on June 22, 2009, for 5 days of training. From June 25 to 26, a total of 59 personnel presented with fever and respiratory symptoms and tested positive. The index patient 


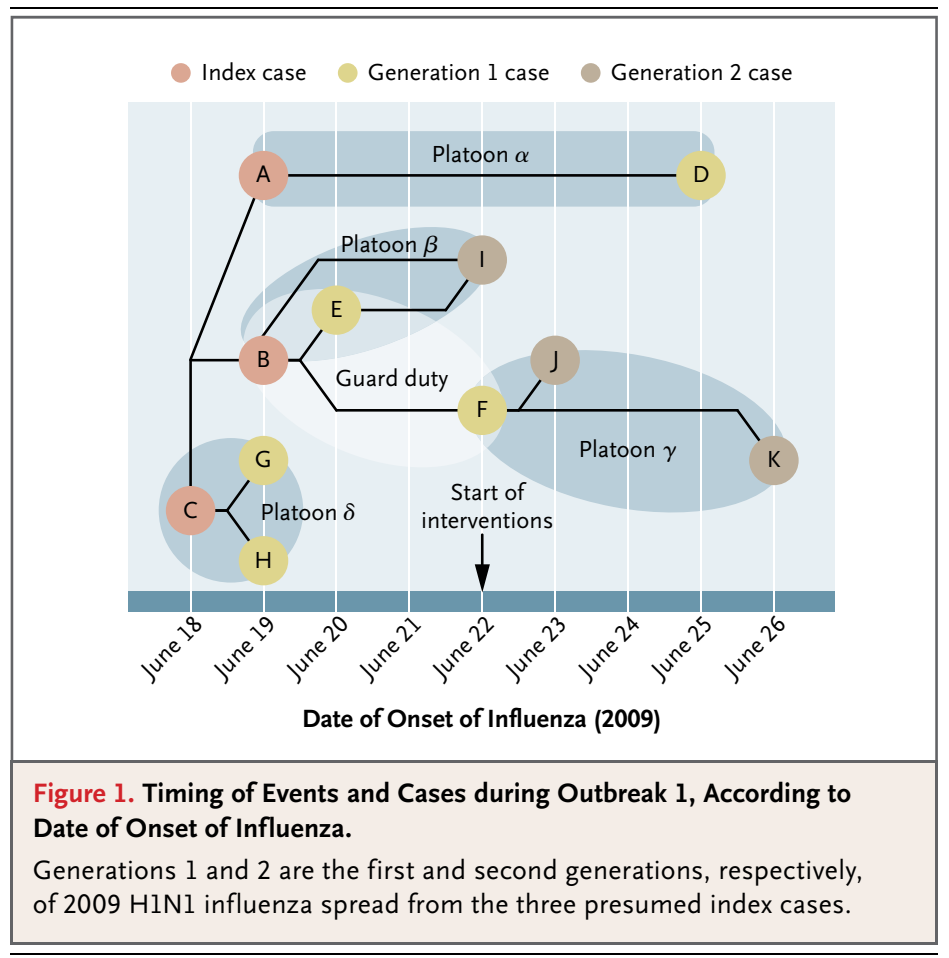

could not be conclusively identified. Prophylaxis was begun in the remaining 634 personnel, who were given home leave after completion of training on June 26. They were followed by means of telephone surveillance. Throughout the outbreak period, a total of 63 personnel $(9.1 \%)$ had confirmed infection (Fig. 2). After prophylaxis was completed, the remaining 630 unaffected personnel were surveyed by means of telephone; 535 (84.9\%) responded, of whom 517 (96.6\%) reported having completed the prophylaxis. A total of 41 respondents reported having respiratory symptoms, and 10 reported having fever with respiratory symptoms. Of these personnel, six and five, respectively, were tested; all tests were negative.

\section{MOLECULAR SEQUENCING}

The use of whole-genome sequencing allowed for a molecular epidemiologic analysis, as previously described. ${ }^{25}$ Whole-genome sequences were used to identify the relatedness of the isolated viruses and to suggest clusters of transmission to further describe the conditions of the outbreak (Fig. 3).

Each of the four outbreaks formed a distinct cluster, with the closest international strains derived from the New York lineage A/New York/18/
2009(H1N1). Outbreak 4 comprised two viral clusters, one New York-like and the other similar to the Singapore local-nightclub cluster ${ }^{24}$; strains isolated during the other outbreaks matched Singapore strains closely. The whole-genome sequences of viruses from outbreak 2 were tightly clustered, suggesting a single causal virus, whereas the local components of outbreaks 1,3 , and 4 were from introductions of highly related Singapore strains, not repeated introductions of distinct viruses. The molecular evidence strongly supports the results of our epidemiologic investigation, which bear out the premise that the outbreaks consisted of transmitted cases of infection rather than unrelated cases.

All seven confirmed cases with an onset after oseltamivir prophylaxis occurred within 4 days after the intervention. The affected patients had complied with the prophylaxis; at the time of infection, they were switched to a treatment dose. In six of the seven cases, there was sufficient genetic material for sequencing. None of the sequenced samples (37 in total, including these 6) had any known or suspected mutations that might have conferred resistance to oseltamivir (including the H274Y mutation).

\section{RATES OF INFECTION AND EFFICACY OF INTERVENTIONS}

The overall proportion of personnel with infection before the oseltamivir prophylaxis and the other interventions were instituted was $6.4 \%$ across all four military units (Table 1). After prophylaxis was begun, in combination with home leave coordination of schedules to avoid contact among the units at the camp, seven more cases were confirmed $(0.6 \%$ of the study population). After intervention, the infection rate was reduced to $5.9 \%$ of the original rate ( $95 \%$ credible interval, 2.5 to 10.9), (posterior hypothesis probability, <0.001).

Guided by the phylogenetic analyses, we used mathematical modeling to investigate the effect of the interventions on the course of the outbreaks. If we considered only confirmed cases, the global estimate of the reproductive number before intervention was 1.91 (95\% credible interval, 1.50 to 2.36). There was a significant reduction in the reproductive number after intervention, to 0.11 ( $95 \%$ credible interval, 0.05 to 0.20 ) (posterior hypothesis probability, <0.001). If untested, symptomatic cases were included, the reproductive 
number before the interventions was 1.85 (95\% credible interval, 1.48 to 2.24), with a significant reduction after intervention, to 0.28 ( $95 \%$ credible interval, 0.20 to 0.38 ) (posterior hypothesis probability, <0.001).

The rate of infection was clearly reduced as a result of interventions in outbreaks 2,3 , and 4 (Table 1). In outbreak 4, ring prophylaxis coincided with the sending home of personnel; thus, to test the effectiveness of prophylaxis, we projected the distribution of one further generation of cases, using the posterior mean of the reproductive number during the preintervention period (Fig. 2). The two distributions we estimated represent what we would expect if the apparent efficacy of the interventions was due to chance alone or due to the isolation measures, not the oseltamivir prophylaxis. The large discrepancy between these distributions and the observed trajectory of the epidemic strongly suggests that the sharp drop in rate of infection was due to prophylaxis, which reduced the transmission of the virus, as well as isolation (rather than isolation alone).

\section{SIDE EFFECTS OF OSELTAMIVIR}

We surveyed a total of 816 personnel for side effects of oseltamivir prophylaxis. In all, 63 (7.7\%) reported mild, nonrespiratory symptoms (Table 2). No neuropsychiatric events or severe adverse events were reported.

\section{DISCUSSION}

Many essential services are provided by persons who work in semiclosed or closed environments where influenza outbreaks can be rapid and severe. ${ }^{6,26}$ In an influenza outbreak among Taiwanese military recruits, the rate of infection was $57.7 \%{ }^{27}$; an influenza A (H3N2) outbreak on a U.S. Navy ship had an infection rate of $42 \% .{ }^{28}$ High rates of infection are also reported at schools, which are similarly enclosed. One boarding school had 56 cases (in $6.5 \%$ of the population) a week after the index case occurred, ${ }^{29}$ and another had an overall rate of infection of $71 \% .{ }^{30}$ During a New York City school outbreak of the 2009 pandemic influenza A (H1N1) virus, 35\% of students reported symptoms of influenza-like illness. ${ }^{31}$ In our study, during outbreak 4, 59 cases occurred within 4 days after the first contact with the index patient.

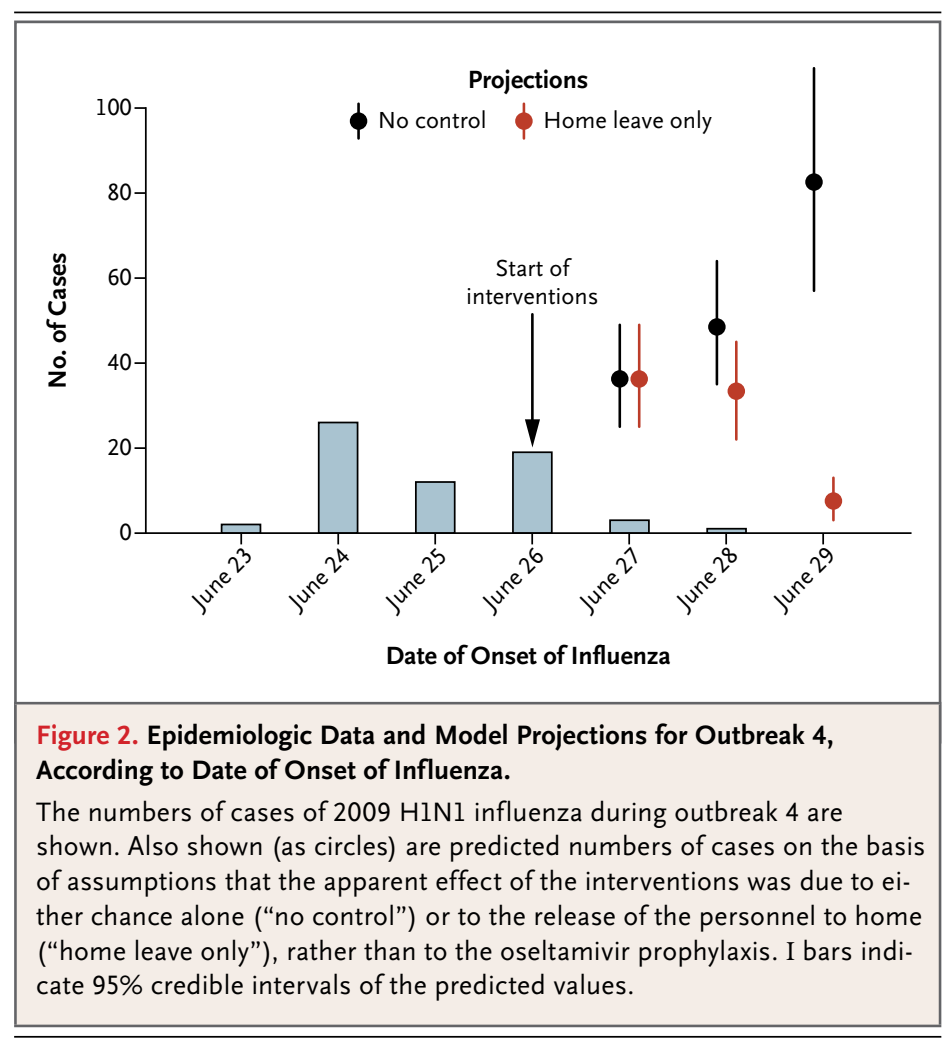

Two modeling studies of the containment of pandemic epicenters, although not specifically based on closed communities, have predicted the effectiveness of ring prophylaxis. ${ }^{4,5}$ The effectiveness of antiviral prophylaxis has not been well documented in outbreak situations outside the household setting. ${ }^{32}$ The use of postexposure prophylaxis with oseltamivir in close household contacts of patients with seasonal influenza resulted in protective efficacies of $68 \% \%^{7}$ and $89 \%{ }^{33}$ against clinically diagnosed influenza. Early prophylaxis with amantadine also reduced the incidence of influenza, and its associated mortality rate, in outbreaks at long-term care facilities. ${ }^{34}$

For the 2009 influenza pandemic, H1N1 observations suggest that antiviral prophylaxis administered in contacts within households, schools, and workplaces is effective in slowing transmission. ${ }^{35}$ In the present study, we have shown that ring prophylaxis with oseltamivir given after exposure in military camps, including a health care setting, was effective, allowing training and operations to continue while substantially reducing the risk of further generations of cases during prophylaxis. The settings studied have the poten- 


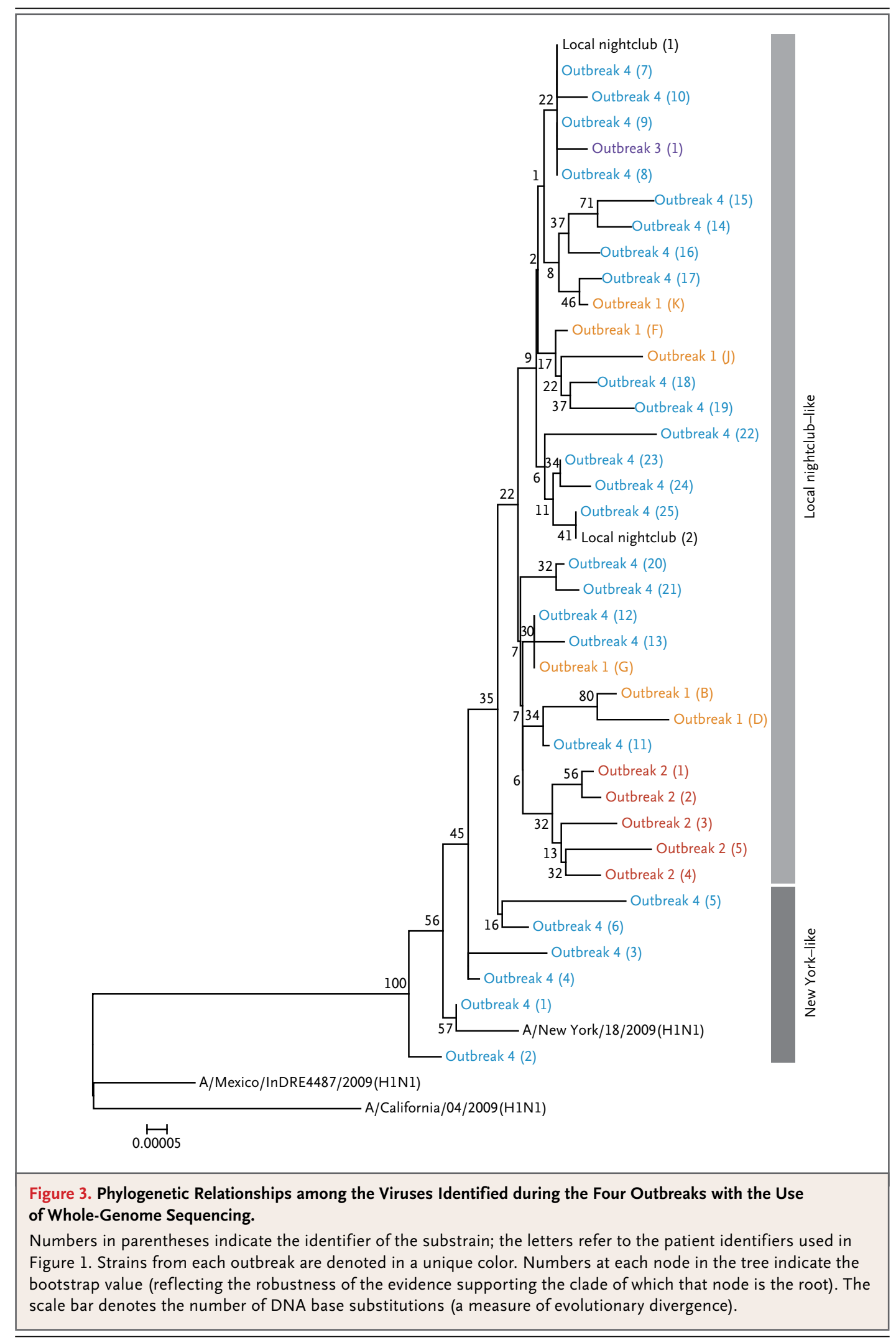


tial for intense transmission and are similar to environments such as hospital wards, boarding schools and other schools, and long-term care facilities. The initial response to outbreak 1 also reflects the limitations of quarantining only people considered to be close contacts of an affected patient, since some cases were identified in patients who were contacts, but not close contacts as defined by the Singapore Ministry of Health. Ring prophylaxis, based on spatial proximity, was more effective in controlling the spread of disease than was an exclusive focus on close contacts.

The pandemic (H1N1) 2009 vaccine is now available $^{36}$; however, antiviral prophylaxis may be considered as an additional strategy in reducing the pandemic's effects, especially in areas in which the supply of vaccine is limited. Furthermore, this strategy may be important in future epidemics and pandemics, either before vaccines are available or when there is a poor match between the vaccine and circulating strains.

The threshold for initiating neuraminidaseinhibitor prophylaxis has not been well defined. For outbreaks 1, 2, and 3 in our study, prophylaxis was initiated early and was followed by rapid cessation of the outbreak. This was possible because of rapid detection through health education, surveillance through daily measurement of temperature and monitoring of symptoms, and laboratory testing. Although outbreak 4 was not detected early, postexposure prophylaxis was effective in breaking the chain of transmission and probably helped prevent a higher rate of infection.

Study limitations include the facts that the data were observational and that multiple interventions were applied simultaneously. The relative strength of the nonpharmaceutical interventions as compared with prophylaxis could only be inferred through modeling. However, it would have been difficult to use prophylaxis as the sole control measure, owing to external pressure to do everything possible to halt transmission and the spontaneous social-distancing measures people take. Although the best efforts were made to ensure consistency of the data collection and use of interventions across the four outbreaks, local circumstances influenced the study activities and should be considered part of any investigation of outbreaks. In addition, monitoring data were incomplete for some outbreaks, because personnel completed their training and were given home

\begin{tabular}{|c|c|}
\hline \multirow[t]{2}{*}{ Side Effect } & $\begin{array}{c}\text { Personnel } \\
(\mathrm{N}=816)\end{array}$ \\
\hline & no. (\%) \\
\hline Diarrhea & $14(1.7)$ \\
\hline Headache & $9(1.1)$ \\
\hline Nausea or vomiting & $22(2.7)$ \\
\hline Dizziness & $5(0.6)$ \\
\hline Epigastric pain & $4(0.5)$ \\
\hline Drowsiness & $8(1.0)$ \\
\hline Mild allergic reaction (rash) & $6(0.7)$ \\
\hline
\end{tabular}

leave; we subsequently performed telephone surveillance instead to obtain as much information as possible.

The use of oseltamivir prophylaxis as a containment measure may be limited to semiclosed or closed communities, since transmission in communities in the general population may subsequently lead to further outbreaks. In the boarding school where the use of amantadine prophylaxis significantly reduced the number of influenza cases, the number of cases increased after the prophylaxis was stopped. ${ }^{29}$ However, the overall rate of infection was significantly lower than expected, and cases were spread out over time, reducing the peak rate of absenteeism.

Our experience provides evidence that early case detection and the use of antiviral ring prophylaxis effectively truncate the spread of infection during an epidemic, giving empirical support to theoretical mathematical models. Aggressive prophylaxis may be justifiable to provide protection from an influenza strain that causes severe disease or to protect vulnerable populations such as frail or elderly residents of long-term care facilities or persons in closed or semiclosed environments such as schools, prisons, and military camps. Finally, containing the pandemic's spread may postpone the onset of substantial illness and distribute temporally the burden on the health care system until other control measures, such as vaccine, become available.

Supported by the Singapore Ministry of Defence, the National University of Singapore (for the statistical analysis), and the Singapore Agency for Science, Research, and Technology (for the genetic sequencing).

Disclosure forms provided by the authors are available with the full text of this article at NEJM.org. 
We thank the officers and personnel of the units in which the outbreaks occurred and the respective medical centers for their participation in the epidemiologic investigation; the DSO National Laboratories for laboratory support; the Singapore Agency for Science, Technology and Research - especially Dr. Sebas- tian Maurer-Stroh and the Bioinformatics Institute (http:// mendel.bii.a-star.edu.sg/METHODS/flumapIntro.html) - for the genetic and bioinformatics analyses; and staff at the Biodefence Centre, Headquarters Medical Corps, and Headquarters Army Medical Services for their help with the study.
REFERENCES

1. Human infection with new influenza A (H1N1) virus: Mexico, update, March-May 2009. Wkly Epidemiol Rec 2009;84:2139.

2. Ministry of Health, Singapore. Influenza pandemic readiness and response plan, updated January 2009. (Accessed May 14, 2010, at http://app.crisis.gov.sg/Data/ Documents/FluPandemicPlan/Main DocumentPublic_Jan09.pdf.)

3. Centers for Disease Control, Department of Health, R.O.C (Taiwan). Influenza pandemic strategic plan, June 2, 2009. (Accessed May 14, 2010, at http://www.cdc gov.tw/public/data/92261185971.pdf?bcsi_ scan_09F03AE3CB5B1BFD=0\&bcsi_scan_ filename $=92261185971$.pdf.)

4. Longini IM Jr, Nizam $\mathrm{A}, \mathrm{Xu} \mathrm{S}$, et al. Containing the pandemic influenza at the source. Science 2005;309:1083-7.

5. Ferguson NM, Cummings DAT, Cauchemez S, et al. Strategies for containing an emerging influenza pandemic in Southeast Asia. Nature 2005;437:209-14.

6. Vynnycky E, Trindall A, Mangtani P. Estimates of the reproduction numbers of Spanish influenza using morbidity data. Int J Epidemiol 2007;36:881-9.

7. Hayden FG, Belshe R, Villanueva C, et al. Management of influenza in households: a prospective, randomized comparison of oseltamivir treatment with or without postexposure prophylaxis. J Infect Dis 2004;189:440-9.

8. Lee VJ, Chen MI. Effectiveness of neuraminidase inhibitors for preventing staff absenteeism during pandemic influenza. Emerg Infect Dis 2007;13:449-57.

9. Statistics Singapore. Key annual indicators. (Accessed May 14, 2010, at http:// www.singstat.gov.sg/stats/keyind.html\# demoind.)

10. Ministry of Health, Singapore. First confirmed case of influenza A (H1N12009) in Singapore, May 27, 2009. (Accessed May 14, 2010, at http://www .moh.gov.sg/mohcorp/pressreleases.aspx? id=21914.)

11. Idem. Confirmed new cases of influenza A H1N1, June 18, 2009. (Accessed May 14, 2010, at http:/www.moh.gov.sg/ mohcorp/pressreleases.aspx?id=22194.)

12. World Health Organization. DG [Director General] statement following the meeting of the Emergency Committee, June 11, 2009. (Accessed May 14, 2010, at http://www.who.int/csr/disease/swineflu/ 4th_meeting_ihr/en/index.html.)
13. Ministry of Health, Singapore. $\mathrm{MOH}$ circular 79/2009: updates on management of influenza A (H1N1-2009), July 1, 2009. (Accessed May 14, 2010, at http://www .sma.org.sg/references/MOH\%20Circular\% 2079-2009\%201Jul09.pdf.)

14. Idem. Case definitions and management of human infection of influenza A (H1N1-2009). (Accessed May 14, 2010, at https://www.hpp.moh.gov.sg/HPP/ contentportlets/hpp/MungoBlobs/380/57/ Definition\%20(Updated\%207\%20Jul\% 2009).pdf.)

15. Idem. MOH circular 44/2009 - alert: influenza A (H1N1-2009), May 7, 2009. (Accessed May 14, 2010, at http://www .sma.org.sg/references/MOH\%20Circular\% 2044-2009\%207May09.pdf.)

16. Interim guidance on antiviral recommendations for patients with novel influenza A (H1N1) virus infection and their close contacts. Atlanta: Centers for Disease Control and Prevention, May 6, 2009.

17. Chan KH, Peiris JS, Lim W, Nicholls JM, Chiu SS. Comparison of nasopharyngeal flocked swabs and aspirates for rapid diagnosis of respiratory viruses in children. J Clin Virol 2008;42:65-9.

18. World Health Organization. CDC protocol of realtime RT-PCR for influenza A (H1N1), 2009. (Accessed May 14, 2010, at http://www.who.int/csr/resources/ publications/swineflu/realtimeptpcr/en/ index.html.)

19. Lee CW, Koh CW, Chan YS, et al. Large-scale evolutionary surveillance of the 2009 H1N1 influenza A virus using resequencing arrays. Nucleic Acids Res 2010. February 25 (epub ahead of print). 20. Tamura K, Dudley J, Nei M, Kumar S. MEGA4: Molecular Evolutionary Genetics Analysis (MEGA) software version 4.0 Mol Biol Evol 2007;24:1596-9.

21. Cauchemez S, Boëlle PY, Donnelly CA, et al. Real-time estimates in early detection of SARS. Emerg Infect Dis 2006;12: 110-3.

22. Lee PM. Bayesian statistics: an introduction. 3rd ed. New York: Wiley, 2009. 23. R Development Core Team. R: a language and environment for statistical computing. Vienna: R Foundation for Statistical Computing, 2008. (Accessed May 14, 2010, at http://www.R-project.org.) 24. Ministry of Health, Singapore. 16 New confirmed cases of influenza A H1N1, June 21, 2009. (Accessed May 14,
2010, at http://app.crisis.gov.sg/InfluenzaA/ Press.aspx?id=17.)

25. Schreiber MJ, Homes EC, Ong SH, et al. Genomic epidemiology of a dengue virus epidemic in urban Singapore. J Virol 2009;83:4163-73.

26. Milne DG, Cruickshank JD. Report of an outbreak of influenza in the Army. BMJ 1949;1:571.

27. Liu PY, Wang LC, Lin YH, Tsai CA, Shi ZY. Outbreak of influenza A and B among military recruits: evidence from viral culture and polymerase chain reaction. J Microbiol Immunol Infect 2009;42:114-21.

28. Earhart KC, Beadle C, Miller LK, et al. Outbreak of influenza in highly vaccinated crew of U.S. Navy ship. Emerg Infect Dis 2001;7:463-5.

29. Davies JR, Grilli EA, Smith AJ, Hoskins TW. Prophylactic use of amantadine in a boarding school outbreak of influenza A. J R Coll Gen Pract 1988;38:346-8. 30. Delbin DJ, Smithson WH. Red "flu": a study of an epidemic in a girls' boarding school in February 1978. J R Coll Gen Pract 1979;29:151-4.

31. Lessler J, Reich NG, Cummings DAT, et al. Outbreak of 2009 pandemic influenza A (H1N1) at a New York City school. N Engl J Med 2009;361:2628-36.

32. Prevention and control of influenza: recommendations of the Advisory Committee on Immunization Practices (ACIP), 2008. MMWR Recomm Rep 2008;57(RR-7):1-60. 33. Welliver R, Monto AS, Carewicz O, et al. Effectiveness of oseltamivir in preventing influenza in household contacts: a randomized controlled trial. JAMA 2001;285: 748-54.

34. Rubin MS, Nivin B, Ackelsberg J. Effect of timing of amantadine chemoprophylaxis on severity of outbreaks of influenza $\mathrm{A}$ in adult long-term care facilities. Clin Infect Dis 2008;47:47-52.

35. Anderson RM. How well are we managing the influenza $\mathrm{A} / \mathrm{H} 1 \mathrm{~N} 1$ pandemic in the UK? BMJ 2009;339:b2897.

36. Production and availability of pandemic (H1N1) 2009 vaccines. Geneva: World Health Organization, October 30, 2009. (Accessed May 14, 2010, at http:// www.who.int/csr/disease/swineflu/ frequently_asked_questions/vaccine_ preparedness/production_availability/en/ index.html.)

Copyright (c) 2010 Massachusetts Medical Society. 